\title{
The cost-effectiveness of screening for gestational diabetes mellitus in primary and secondary care in the Republic of Ireland
}

\author{
Andriy Danyliv ${ }^{1,2} \cdot$ Paddy Gillespie $^{1} \cdot$ Ciaran O'Neill $^{1}$ - Marie Tierney ${ }^{2,3}$. \\ Angela O'Dea $^{2,3}$ • Brian E. McGuire ${ }^{4,3}$ - Liam G. Glynn ${ }^{5,3}$ - Fidelma P. Dunne .,3 $^{2,3}$
}

Received: 25 June 2015 / Accepted: 10 November 2015 /Published online: 15 December 2015

(C) Springer-Verlag Berlin Heidelberg 2015

\begin{abstract}
Aims/hypothesis The aim of the study was to assess the cost-effectiveness of screening for gestational diabetes mellitus (GDM) in primary and secondary care settings, compared with a no-screening option, in the Republic of Ireland.

Methods The analysis was based on a decision-tree model of alternative screening strategies in primary and secondary care settings. It synthesised data generated from a randomised controlled trial (screening uptake) and from the literature. Costs included those relating to GDM screening and treatment, and the care of adverse outcomes. Effects were assessed in terms of quality-adjusted life years (QALYs). The impact of the parameter uncertainty was assessed in a range of sensitivity analyses.

Results Screening in either setting was found to be superior to no screening, i.e. it provided for QALY gains and cost
\end{abstract}

Electronic supplementary material The online version of this article (doi:10.1007/s00125-015-3824-0) contains peer-reviewed but unedited supplementary material, which is available to authorised users.

\section{Andriy Danyliv}

danyliv@gmail.com

1 J. E. Cairnes School of Business and Economics, National University of Ireland Galway, H91TK33 Galway, Ireland

2 School of Medicine, Clinical Sciences Institute, National University of Ireland Galway, Galway, Ireland

3 Galway Diabetes Research Centre, National University of Ireland Galway, Galway, Ireland

4 School of Psychology, National University of Ireland Galway, Galway, Ireland

5 Discipline of General Practice, National University of Ireland Galway, Galway, Ireland savings. Screening in secondary care was found to be superior to screening in primary care, providing for modest QALY gains of 0.0006 and a saving of $€ 21.43$ per screened case. The conclusion held with high certainty across the range of ceiling ratios from zero to $€ 100,000$ per QALY and across a plausible range of input parameters.

Conclusions/interpretation The results of this study demonstrate that implementation of universal screening is cost-effective. This is an argument in favour of introducing a properly designed and funded national programme of screening for GDM, although affordability remains to be assessed. In the current environment, screening for GDM in secondary care settings appears to be the better solution in consideration of costeffectiveness.

Keywords Cost-effectiveness · Costs and cost analysis . Cost-utility analysis $\cdot$ Economic evaluation $\cdot$ Gestational diabetes mellitus $\cdot$ Screening

$\begin{array}{ll}\begin{array}{l}\text { Abbreviations } \\ \text { ATLANTIC DIP }\end{array} & \text { Atlantic Diabetes in Pregnancy } \\ \text { CEAF } & \text { Cost-effectiveness acceptability frontier } \\ \text { GDM } & \text { Gestational diabetes mellitus } \\ \text { GP } & \text { General practitioner } \\ \text { HIQA } & \text { Health Information and Quality Authority } \\ \text { IADPSG } & \text { International Association of the } \\ & \text { Diabetes and Pregnancy Study Groups } \\ \text { NICU } & \text { Neonatal intensive care unit } \\ \text { NMB } & \text { Net monetary benefit } \\ \text { QALY } & \text { Quality-adjusted life year } \\ \text { WTP } & \text { Willingness to pay }\end{array}$




\section{Introduction}

Gestational diabetes mellitus (GDM) is defined as any degree of glucose intolerance with onset or first recognition during pregnancy [1] and is associated with maternal and perinatal complications, as well as additional healthcare resource utilisation and costs [2-6]. While international GDM prevalence estimates vary considerably, partly due to differences in diagnostic criteria [7], it is widely recognised that GDM prevalence is rising worldwide $[1,8]$, leading to increasing pressures being placed on already constrained healthcare budgets. As a result, and given established evidence that treatment of GDM substantially reduces the risk of maternal, perinatal and neonatal complications [9-12], screening strategies to effectively identify and treat GDM are being implemented in many countries. A number of economic evaluations based on decision modelling techniques have demonstrated the costeffectiveness of various screening strategies, in jurisdictions including the USA, UK, India and Israel [13-17]. Despite modest expected average effects on delivery outcomes [18], the relatively low cost of screening and subsequent treatment provides for favourable incremental cost-effectiveness of these screening strategies given treatment compliance after diagnosis. Notably, however, in all of the mentioned modelling studies the issue of screening uptake was not considered directly.

In Ireland, GDM diagnosed using the criteria of the International Association of the Diabetes and Pregnancy Study Groups (IADPSG) affects $12.4 \%$ of all pregnancies [5]. No national GDM screening programme is currently in operation, and existing practice may be best characterised as opportunistic in nature. In 2007, the Atlantic Diabetes in Pregnancy (ATLANTIC DIP) project was established in five hospital settings along the Irish Atlantic seaboard, promoting a strategy to offer all pregnant women universal screening for GDM in a hospital setting [5]. Screening involved a $2 \mathrm{~h}$ OGTT with a $75 \mathrm{~g}$ glucose load. Following low screening uptake, however, it was considered that providing screening for GDM in the primary care setting, which offers wider geographical access to patients, might yield superior screening uptake [19]. A randomised controlled trial was conducted to test this hypothesis, but, contrary to expectations, a smaller uptake was observed in the primary care setting relative to the secondary care setting: $52.69 \%$ vs $89.23 \%$ as assessed on a 'per protocol' basis [20]. Partly this might be attributed to women's perceptions of higher quality care, superior expertise and comprehensive antenatal care in the hospital setting [21], and partly to considerations of additional workload for general practitioners (GPs) [20]. This notwithstanding, a further question of interest is that of the relative costeffectiveness of GDM screening in primary vs secondary care.

We undertook an economic evaluation of screening for GDM in Ireland based on a decision analytical model. We considered the alternative strategies of screening in primary and secondary care, and compared these to the no-screening alternative, as well as to each other. In the decision model, alternative screening strategies assumed different uptake rates, which consequently led to different proportions of GDM cases being detected and treated. We incorporated data from a range of sources: Irish-specific data on pregnancy outcomes with and without GDM, and international data on the effect of GDM treatment on such outcomes. In terms of costs, the model estimated those relating to GDM screening, GDM treatment and the management of adverse outcomes. In terms of effectiveness, the model estimated quality-adjusted life years (QALYs). Uncertainty in cost per QALY estimate was examined in the model using probabilistic sensitivity analyses. In the base case analysis, the alternative screening strategies were modelled on the uptake rates observed in the randomised controlled trial [20]. Other scenarios were assessed in one- and two-way sensitivity analyses, varying uptake rates and other key parameters across their plausible ranges.

\section{Methods}

For the economic evaluation, we followed the Health Information and Quality Authority (HIQA) guidelines for the Republic of Ireland [22]. These informed the adoption of the healthcare perspective (publicly funded system) with respect to costing (in 2013 euro prices), the cost-utility analysis framework for evaluation (QALY as the measure of effect), and the use of a $5 \%$ discount rate in the base case. The alternative options considered in the model were: (1) screening at a GP practice (primary care); and (2) hospital-based screening (secondary care). Given that little is known of the scale of opportunistic screening for GDM currently in place in Ireland, we considered a no-screening option as a comparator in order to explore the cost-effectiveness in the full continuum of screening uptake. Screening was based on a $75 \mathrm{~g}$ OGTT assumed to have $100 \%$ sensitivity and specificity, as this is the reference test [16]. It was assumed that diagnosis would be followed by treatment in the form of dietary advice and insulin therapy if necessary.

The decision model The evaluation was based on the analytical decision-tree model built for this study (Fig. 1). The model structure was informed by similar models from other settings [13-18] and by observational data from the large-scale ATLANTIC DIP study conducted in the west of Ireland [5] on the risks of maternal, neonatal and perinatal outcomes associated with GDM in the Irish context. Complications entered the model if their risks had been shown to be altered by GDM or its treatment, and if they were expected to have cost or qualityof-life implications [10, 11]. The model structure, presented and described in Fig. 1, was validated by a panel of clinical 


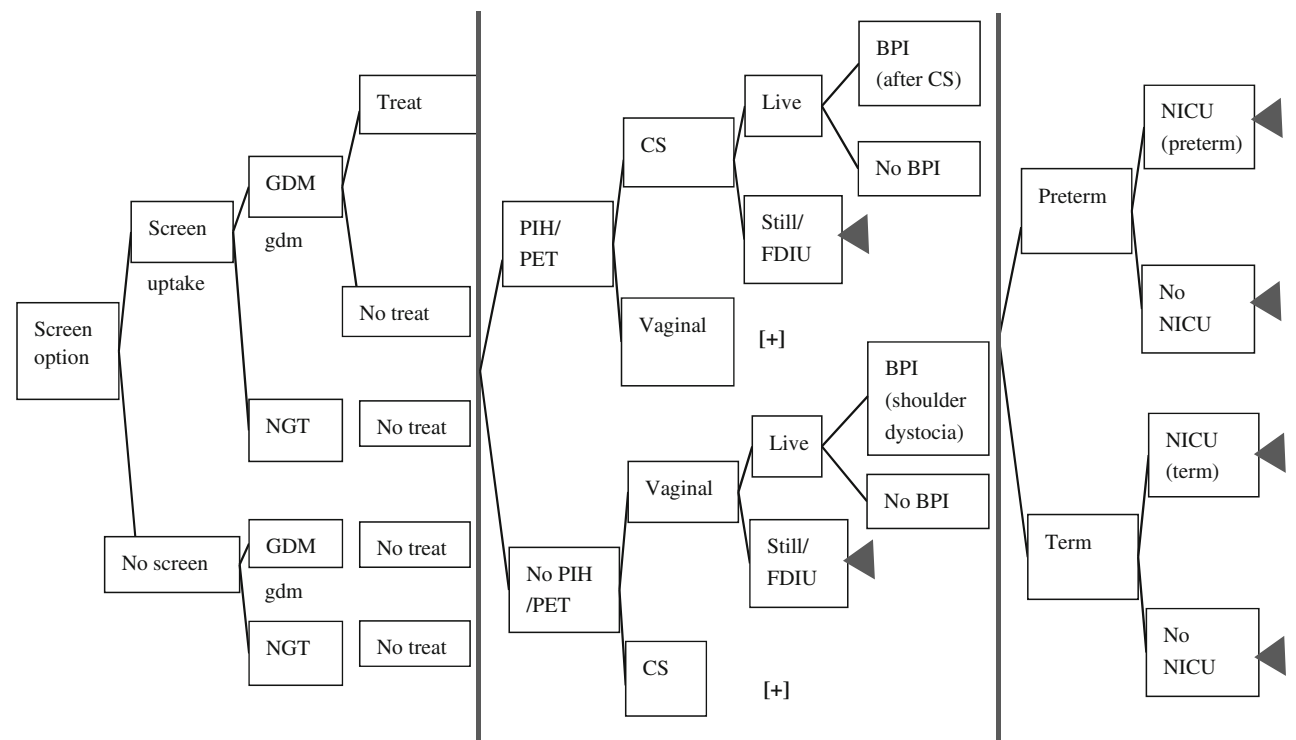

Fig. 1 The decision-tree model structure. The model is collapsed to the representative branches for the convenience of presentation (the collapsed branches are represented by $[+]$ ). The terms outside the boxes, i.e. 'uptake' and 'gdm', show the names of the parameters used in the decisiontree modelling. Maternal outcomes consisted of hypertensive disorders in pregnancy (pregnancy-induced hypertension $[\mathrm{PIH}]$ or pre-eclamptic toxaemia [PET]) and the mode of delivery (Caesarean section [CS] or vaginal), the latter also conditional on the former. Neo- and perinatal

experts specialising in endocrinology and obstetrics. It attempted to capture long-term (lifetime) effects of the complications. The parameters used to populate the model are presented in the electronic supplementary material (ESM Table 1) and are described below.

Risks and treatment effects As discussed, we built upon a previously conducted trial reporting $52.69 \%$ uptake in the primary care setting and $89.23 \%$ in the secondary care setting [20]. The treatment uptake after GDM detection was set to be $90 \%$ in both primary and secondary care groups for the base case [18]. The risk of GDM was set to be $12.36 \%$ in the base case, as reported in the ATLANTIC DIP study (680 out of 5,500 pregnancies) [5]. The baseline risks of complications in the group with normal glucose tolerance and with treated GDM were based on the data from the ATLANTIC DIP study [5], deemed to be the best estimates for the Irish population. In order to obtain the risks that would have occurred in the group with untreated GDM, we used the results of the multicentre randomised controlled trial from the USA reported by Landon et al [12], due to the fact that this study applied criteria for diagnosis of mild GDM that are close to the IADPSG criteria used in our study. Available systematic reviews $[10,11]$, both incorporating the study by Landon et al [12], combine evidence for GDM diagnosed on various criteria, but still report very similar treatment effects. outcomes included brachial plexus injury (BPI) (shoulder dystocia after vaginal delivery, and own risk after Caesarean section) and prematurity as separate conditions having potentially lasting effects. After being born prematurely a baby was estimated (ATLANTIC DIP data) to have a longer neonatal intensive care unit (NICU) stay than would have occurred for other reasons. NGT, normal glucose tolerance; FDIU, fetal demise in utero

Costs The costs of screening and contacting women in primary and secondary care settings were assessed via microcosting in compliance with HIQA guidelines (ESM Table 2) [22]. The direct costs of screening in primary and secondary care were not different, due to a similar level of staff involvement and very similar procedures in each setting. However, we anticipated higher overheads in the secondary care setting. Therefore, a secondary to primary care cost ratio of 1.174 was applied (an average cost of an outpatient procedure in hospital to the cost of an average $30 \mathrm{~min}$ primary care procedure in the UK, according to Personal Social Services Research Unit data [23]). Thus, primary care screening was assigned a baseline cost estimate of $€ 35$ and secondary care screening $€ 41.09$ in the base case.

Other cost parameters that entered the model were taken from the published literature, inflated to 2013 prices using country consumer price index data and transferred to Ireland using purchasing power parity indexes [24]. The cost of treating GDM in Ireland was previously assessed by Gillespie et al [25] and was $€ 506.36$ in 2014 prices. Other Irish-specific costs were assigned to the cost of delivery (vaginal delivery $€ 2,588.13$, Caesarean delivery $€ 6,287.64$ ) and stay in the neonatal intensive care unit (NICU) for term and premature babies: $€ 4,102.87$ and $€ 17,086.28$, respectively (cost of 1 day $€ 899.75$ ). These figures were previously reported by Gillespie et al [25] based on Casemix data (Department of Health and Children) [26]. The cost of hypertensive disorders in pregnancy was assessed to be $€ 7,840$ : an average of the 
incremental estimates provided by the two studies from the USA and Israel [27, 28]. Other studies providing nonincremental and, consequently, higher estimates were disregarded [29, 30]. The incremental cost of a stillbirth (including fetal demise in utero), compared with that of a live birth, was assessed to be $€ 2,055$, based on a study from the UK providing the most comprehensive costing [31]. The incremental costs of a premature baby were estimated to be $€ 2,064$, based on a UK study, after subtracting neonatal and delivery costs to avoid double-counting [32]. Finally, the cost of brachial plexus injury in a newborn was assigned $€ 14,540$, based on the weighted average estimates from the USA of the lifetime cost for moderate to severe cases [33].

QALYs The effectiveness of screening was measured in QALYs discounted over the lifetime. Maternal life expectancy was set at 51 years after delivery, the baby's life expectancy was set at 81 years [34], and these were not varied. The baseline quality of life with no complications was set as 1 for both mother and baby. We have not identified any evidence of a significant impact of hypertensive disorders in pregnancy on quality of life. Stillbirth was set to have a lifetime impact on the mother, reducing quality of life to 0.92 [35]. The QALYs over the full life expectancy of a baby were lost due to stillbirth. Premature birth was set to reduce maternal quality of life by 0.0452 to 0.9548 in the first year only [36], and the baby's quality of life by 0.04 to 0.96 for the whole lifetime [17], although evidence for the latter is conflicting. There was no evidence of a permanent effect of brachial plexus injury on maternal quality of life, but the baby's quality of life was reduced to 0.6 for the full lifetime [33].

Sensitivity analyses The base case, i.e. the expected case with the values of the parameters that are most likely to occur, was assessed in a probabilistic manner. This implied running 10,000 Monte Carlo simulations where the parameters were varied according to the assigned distributions (based on the parameters reported in the sources), as described in ESM Table 1: beta distributions for risks, log-normal for treatment effects, gamma for cost of health conditions. The cost parameters obtained from micro-costing were varied uniformly in a range between

Table 1 Results of the base case analysis. Deterministic analysis uses the point estimates for the input parameters. Probabilistic analysis is based on 10,000 Monte Carlo simulations and presents the summary for the
$+/-20 \%$ of the deterministic estimate, as per HIQA guidelines [22]. Based on the simulated values of the incremental costs and effects, cost-effectiveness acceptability frontiers (CEAFs) were built to calculate the probability of the three screening options being cost-effective for willingness to pay (WTP) per QALY (or ceiling ratio) ranging from $€ 0$ to $€ 100,000$.

This was followed by one-way sensitivity analyses of the four parameters: the discount rate varied from $0 \%$ to $6 \%$ according to HIQA recommendations [22]; the secondary to primary care cost ratio varied from 0.9 to 1.4 (1.17 being approximately in the middle); and the two treatment uptake rates varied from $80 \%$ to $100 \%$. In order to work across different combinations of the uptake rates in secondary and primary care settings, a two-way sensitivity analysis was performed, changing the screening uptake in both settings across the plausible range between $40 \%$ and $100 \%$. This was done deterministically, due to the high computational burden of probabilistic simulations. Probabilistic one-way sensitivity analysis was confined to the ratio of the cost of screening between secondary and primary care settings. Here the value of the parameter was varied from 0.9 to 1.4 (in steps of 0.005 ), and 200 simulations for each value were run $(20,000$ draws in total). The results of the base case and sensitivity analyses were compared with the ceiling ratio at the two HIQA recommended levels: €20,000 and €45,000 per QALY.

\section{Results}

Base case The results of the base case (Table 1) demonstrate that both screening options are dominant (more effective and less costly) in comparison with no screening, and secondary care screening dominates over primary care screening. This can also be seen on the cost-effectiveness plane (Fig. 2a), where both options are in the dominant quadrant, and the mean and deterministic value for hospital screening options lies to the south-east of the GP screening option. The probability of the hospital option being dominant stays high on the whole region of the ceiling ratios from $€ 0$ to $€ 100,000$, as presented on the CEAF (Fig. 2b). In the HIQA recommended region between $€ 20,000$ and $€ 45,000$ per QALY, hospital-based screening has

incremental cost-effectiveness ratio (ICER) based on the ratio of expected incremental costs and effects

\begin{tabular}{|c|c|c|c|c|c|c|c|c|}
\hline \multirow[t]{2}{*}{ Option } & \multicolumn{2}{|l|}{ Total } & \multicolumn{3}{|c|}{ Incremental (to no screening) } & \multicolumn{3}{|c|}{ Incremental (to previous) } \\
\hline & Cost $(€)$ & QALY & Cost $(€)$ & Effect & ICER & Cost $(€)$ & Effect & ICER \\
\hline No screen & $5,395.95$ & 37.7746 & - & - & - & - & - & - \\
\hline GP & $5,358.23$ & 37.7754 & -37.72 & 0.0008 & Dominant & - & - & - \\
\hline Hospital & $5,336.80$ & 37.7759 & -59.15 & 0.0014 & Dominant & -21.43 & 0.0006 & Dominant \\
\hline
\end{tabular}

Base case implies screening uptake $52.69 \%$ in primary care and $89.23 \%$ in secondary care 


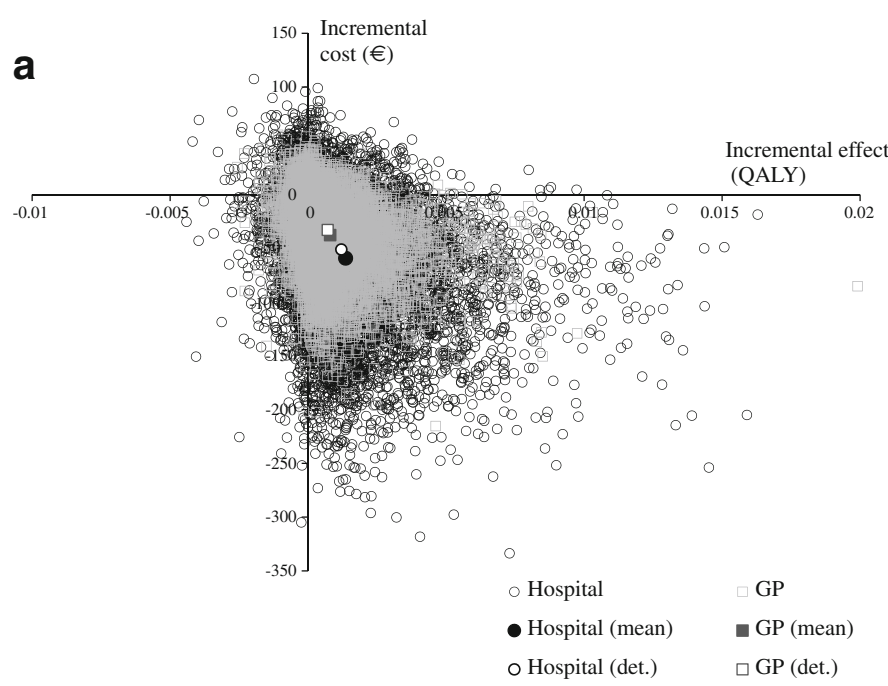

Fig. 2 Results of the base case analysis. (a) Cost-effectiveness plane. (b) CEAF: hospital-based screening (grey shaded area) is the dominant alternative over the wide range of WTP values. Other options (GP

the highest probability of being cost-effective, i.e. between $86.5 \%$ and $87 \%$ depending on the threshold.

One-way sensitivity analyses The one-way sensitivity analyses for the four fixed input parameters, i.e. discount rate, hospital to GP practice-based cost of screen ratio and treatment uptake rates in both settings, based on deterministic estimations, did not alter the results: hospital-based screening yielded higher expected effects and lower expected costs than primary care screening throughout the plausible range of all input parameters. These results are not presented graphically, as the hospital-based alternative always dominated GP practice-based screening. Changing the secondary to primary cost of screen ratio between 0.9 and 1.4 (with 200 probabilistic runs for each value) did not change the results either, and screening in the secondary care setting remained the most cost-effective option: the likelihood of being cost-effective ranged from $94 \%$ to $79 \%$ for the ceiling ratio of $€ 20,000$ per QALY and from $89.5 \%$ to $82 \%$ for $€ 45,000$ per QALY (Fig. 3).

Two-way sensitivity analysis of screening uptake The twoway sensitivity analysis in which the screening uptake rates were varied deterministically across the plausible range of $40 \%$ to $100 \%$ is presented in Fig. 4. It represents the difference in net monetary benefit (NMB) between hospital-based screening and GP practice-based screening for a ceiling ratio of $€ 20,000$ (Fig. 4a) and $€ 45,000$ (Fig. 4b). The border of zero NMB divides the set of uptake values into regions where primary care screening is most cost-effective (above the line) and where secondary care screening is most cost-effective (below the line). Thus, in the higher range of uptake (around $90 \%-100 \%$ ), for hospital-based screening to remain costeffective its screening uptake should exceed that in primary care by no less than 8.8 percentage points if the ceiling ratio is

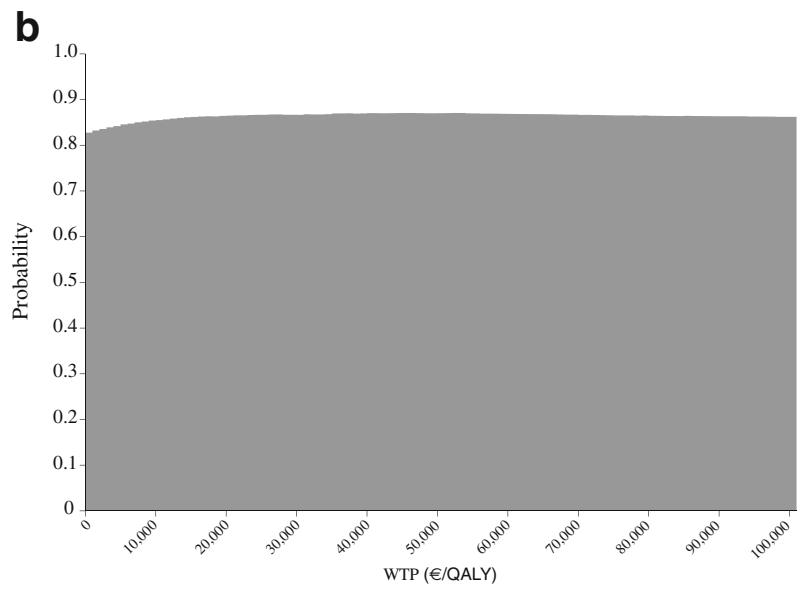

practice-based or no screening) do not appear at the CEAF as they are not cost-effective. det., deterministic value

$€ 20,000$ per QALY, or by no less than 6.6 percentage points if the ceiling ratio is $€ 45,000$ per QALY. In the base case this difference is 36.5 percentage points.

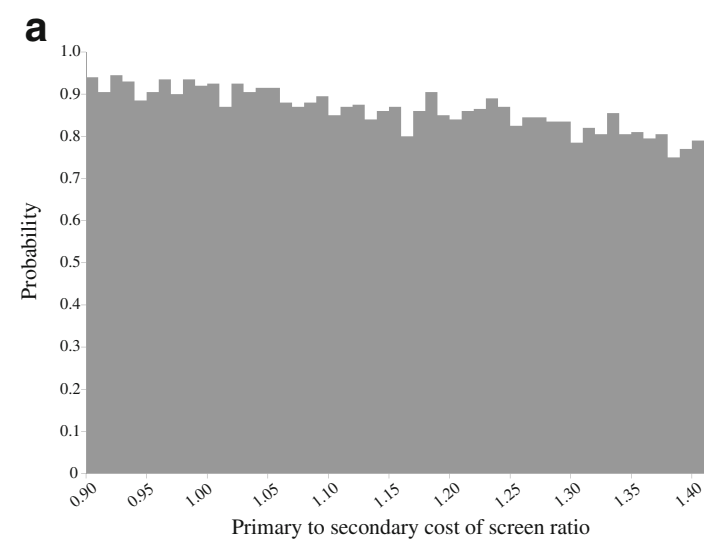

b

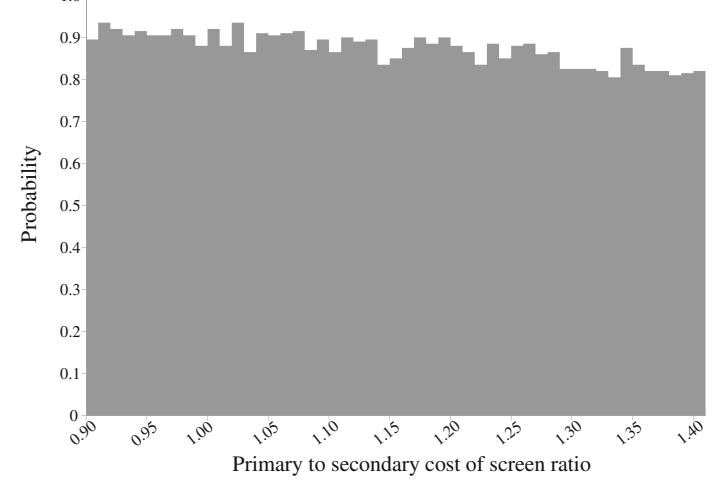

Fig. 3 CEAF: one-way sensitivity analysis of the primary to secondary cost of screen ratio with probabilistic runs (a) for WTP $€ 20,000$; (b) for WTP $€ 45,000$. Hospital-based screening (grey shaded area) is a dominant option over the plausible range of the primary to secondary cost of screen ratio. Other options (GP practice-based or no screening) do not appear at the CEAF as they are not cost-effective 
a

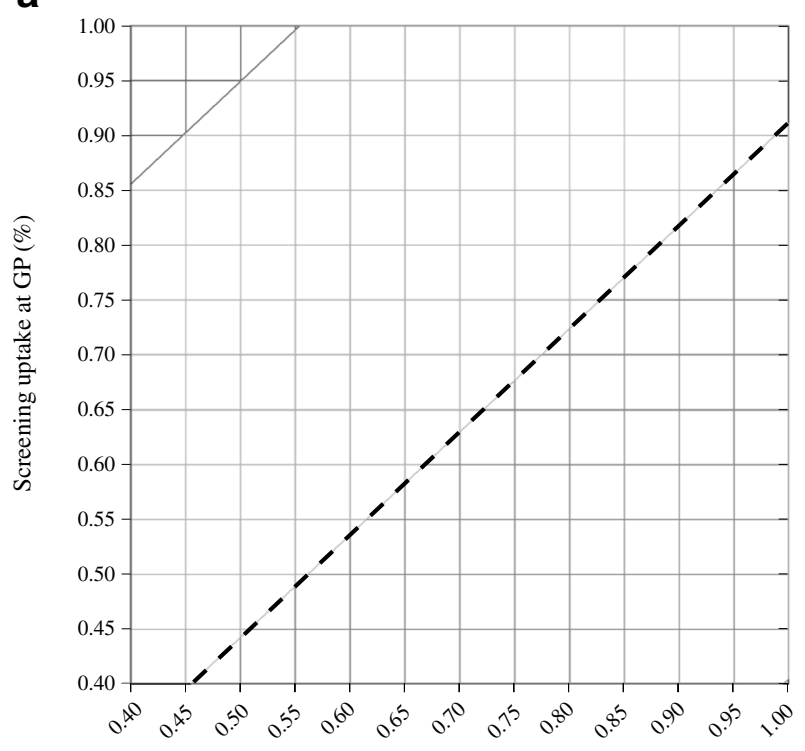

Screening uptake in hospital (\%)

b

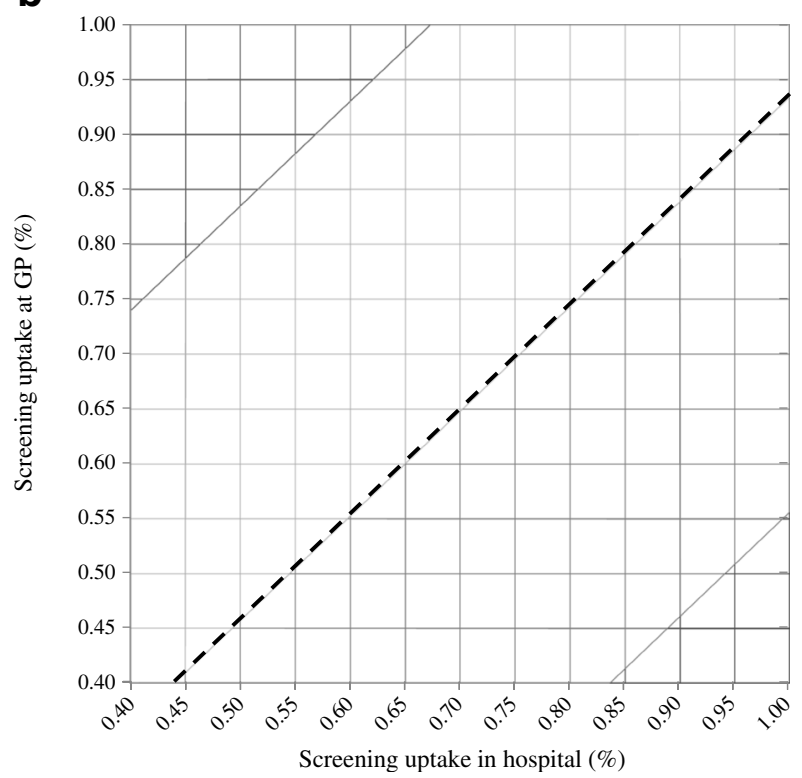

Fig. 4 Two-way sensitivity analyses of the screening uptake rates (a) for WTP $€ 20,000$; (b) for WTP $€ 45,000$. The dashed line represents a set of combinations of screening uptake in hospital and in general practice which corresponds to zero NMB. Above the line is an area where GP screening is cost-effective; below is the area where screening in hospital is cost-effective

\section{Discussion}

We found that screening for GDM is cost-effective compared with no screening, and this conclusion holds with high certainty across the whole range of plausible values of the parameters in our model. This is an argument in favour of a funded national programme of screening for GDM, although affordability remains to be assessed. This is in line with the international literature on the issue of cost-effectiveness of screening for GDM [13-17]. It should be noted that the expected QALY gains are modest, i.e. 0.0008-0.0014 per screened case; the results echo those in outcome modelling studies [18]. But the gains are coupled with expected savings of up to $€ 59$ per screened case arising from better pregnancy outcomes, resulting in screening being superior to no screening.

To our knowledge, the choice between primary and secondary care settings for the provision of screening for GDM has not previously been considered in terms of cost-effectiveness. The base case analysis as well as all the sensitivity analyses point to the secondary care setting being superior to the primary care setting (providing for modest QALY gains of 0.0006 and savings of $€ 21.43$ per case screened). This conclusion holds if the gap between the screening uptake rates is no less than 6.6-8.8 percentage points in favour of secondary care, depending on the ceiling ratio (it is 36.5 percentage points in the base case). These results are also complemented by the qualitative findings from the trial which suggest that, despite distance being an important factor in the decision to attend for screening by women who live far from the hospital, hospital settings are nevertheless preferred to general practice if the distance is equal, due to perception of higher quality care and greater familiarity with secondary care as a source of antenatal care provision [21]. In addition, there were logistical and workload issues experienced among GPs in providing for expanded antenatal care that impacted adversely on this arm of the study, making hospital a more suitable place for screening provision [20].

There is a possibility that GP practice-based screening could operate relatively more cost-effectively if screening in primary care were introduced at a national level. Moreover, if the intention-to-treat cohort are considered, in which the proportion of women who switched screening location in the trial are included, this yields $85.17 \%$ (333 out of 391) uptake in primary care and $91.54 \%$ (357 out of 390) in secondary care, with only a 6.4 percentage point difference between them (based on data from the trial [37]). However, such scenarios are unlikely to represent what would naturally happen in any screening programme on a national scale, given the artificial nature of the intention-to-treat analysis in this particular trial. First of all, switching providers implied different mechanisms and obstacles, and hence cost, that would not be the same under a national programme. Second, increasing uptake in primary care is unlikely to occur without additional incremental cost relative to secondary care, i.e. training and incentivising GP practices, especially given apparent supply shortage [38]. This would shift the secondary to primary cost of screen ratio downwards, again bringing it into the region where hospital is a 
favoured alternative. Additionally, the issue of the quality of care perceptions among users favouring hospital might remain [21].

That said, we did conduct a separate intention-to-treat analysis and the findings still favoured hospital-based screening (results available upon request). In short, it shows that, if such high uptake rates are considered in the primary and secondary locations, hospital-based screening would be costeffective at the lower recommended ceiling ratio of $€ 20,000$ if the cost of screening in secondary care exceeds that in primary care by no more than $12 \%$. Even if the cost excess stays as high as $17.4 \%$, as in the base case (which is very unlikely), hospital-based screening still has an expected incremental cost-effectiveness ratio of $€ 32,300$ per QALY, which is still under the higher threshold of $€ 45,000$.

Strengths and limitations In this study, a model-based approach was selected in order to synthesise available evidence on the risks associated with GDM and potential benefits from its treatment. The choice of the sources for this evidence is driven by the desire to make the model more realistic and reflective of the actual and hypothetical context of universal screening for GDM in the Republic of Ireland. Therefore, the baseline risks were selected from a large observational study conducted in Ireland [5] rather than from systematic reviews which are not specific to this context. The same logic informed the choice of treatment effect from the study conducted in the USA by Landon et al [12]. In that study, mild GDM was diagnosed based on similar criteria to those of the IADPSG applied in our study. In any case, the treatment effects were not substantially different from those reported in the systematic reviews $[10,11]$. On the other hand, the baseline risks in the study of Landon et al [12] differed substantially from those observed in Ireland. This might be explained by very different ethnic composition of the studied groups in the USA [12] from those observed in Ireland [5]. This might also imply that in Ireland treatment effects would be different; however, we do not have any information on this, and the effects reported in the study of Landon et al [12] were taken as the best estimates.

There are several limitations inherent to the model itself. Given the Irish-specific focus of the study, the model populated with the current parameters is limited to Ireland. Nonetheless, the model as a structure can be easily adjusted to other contexts if respective local parameters are provided. In this model, we did not consider long-term effects, such as type 2 diabetes in mothers and children, autistic disorders and cardiovascular diseases. There is a growing body of literature demonstrating associations of GDM or the baby being large for gestational age (the risk of which is also altered by GDM management) with those outcomes [39-45]. While GDM confers an increased risk of these conditions, we have not identified evidence to link the treatment of GDM to reduction of risk of these long-term outcomes. Their inclusion, however, might substantially change the results in favour of the screening option with the higher uptake.

Having no screening rather than the current practice of opportunistic screening in hospital settings as a comparator might be seen as a limitation which might result in overestimation of screening cost-effectiveness. However, our model demonstrates that savings from averting the adverse outcomes of the detected cases outweigh the cost of screening. Thus, every additional screen, given the same (secondary care) or lower cost (primary care), is expected to bring net savings. By this logic, any screening programme increasing screening uptake over that in opportunistic practice is expected to be superior in terms of cost-effectiveness.

Finally, the mixed strategies, where women are able to freely select screening in general practice or in hospital, or where different strategies are offered for different areas, were not directly considered in the model. This is due to the fact that it is impossible to predict what the rates of uptake would be, as current evidence comes from a trial which only considered compulsory randomisation to one location. Moreover, this would also require different arrangements for the administration of such a programme and steering women through the providers of their choice, which implies a different cost structure. Thus, consideration of the mixed strategies would require additional data.

Implications Screening for GDM in Ireland is currently conducted in an opportunistic manner and its extent varies considerably across regions. The results of this study demonstrate that implementation of universal screening is cost-effective. By increasing the detection and management of GDM, such screening will prevent certain cases of adverse maternal and perinatal outcomes, subsequently reducing health resource utilisation and gaining additional QALYs.

In the current environment, screening for GDM in secondary care settings appears to be the better solution both out of consideration of cost-effectiveness and also due to familiarity of both staff and patients with the secondary care setting as a source of antenatal care. Improvements in proximity brought by screening in primary care may provide for only a modest improvement in screening uptake [19] and seem to be outweighed by public preference for the secondary care setting, and operational obstacles in primary care [20,21]. Primary care is unlikely to perform substantially better relative to secondary care without additional cost [38] and thus is unlikely to become a cost-effective alternative under current conditions.

\section{Compliance with ethical standards}

Funding The study was funded by the Health Research Board of Ireland, grant agreement no. ICE/2011/3.

Duality of interest The authors declare that there is no duality of interest associated with this manuscript. 
Contribution statement $\mathrm{AD}$ worked on data analysis, wrote the manuscript, and is a guarantor. PG and CON worked on data analysis and wrote the manuscript. AOD and MT participated in the design of the study, worked on data collection, participated in the discussion of the manuscript at each stage, reviewed/edited it, and provided information and explanation related to the medical part of the data. CON, BEM, LGG and FPD conceived and designed the study, revised all the interim drafts of the manuscript and reviewed/edited them. FPD participated in data analysis and reviewed/edited the manuscript at every stage. All authors saw and approved the final version of this article.

\section{References}

1. The Lancet (2008) The global challenge of diabetes. Lancet 371: 1723

2. Bellamy L, Casas J-P, Hingorani AD, Williams D (2009) Type 2 diabetes mellitus after gestational diabetes: a systematic review and meta-analysis. Lancet 373:1773-1779

3. Danyliv A, Gillespie P, O'Neill C, et al (2015) Short- and long-term effects of gestational diabetes mellitus on the healthcare cost: a cross-sectional comparative study in the ATLANTIC DIP cohort. Diabet Med 32:467-476

4. Gillespie P, Cullinan J, O'Neill C, Dunne F (2013) Modeling the independent effects of gestational diabetes mellitus on maternity care and costs. Diabetes Care 36:1111-1116

5. O'Sullivan EP, Avalos G, O'Reilly M, Dennedy MC, Gaffney G, Dunne F (2011) Atlantic Diabetes in Pregnancy (DIP): the prevalence and outcomes of gestational diabetes mellitus using new diagnostic criteria. Diabetologia 54:1670-1675

6. Wendland EM, Torloni MR, Falavigna M et al (2012) Gestational diabetes and pregnancy outcomes - a systematic review of the World Health Organization (WHO) and the International Association of Diabetes in Pregnancy Study Groups (IADPSG) diagnostic criteria. BMC Pregnancy Childbirth 12:23

7. Reece EA, Leguizamón G, Wiznitzer A (2009) Gestational diabetes: the need for a common ground. Lancet 373:1789-1797

8. Ferrara A (2007) Increasing prevalence of gestational diabetes mellitus: a public health perspective. Diabetes Care 30(Suppl 2): S141-S146

9. Crowther CA, Hiller JE, Moss JR, McPhee AJ, Jeffries WS, Robinson JS (2005) Effect of treatment of gestational diabetes mellitus on pregnancy outcomes. N Engl J Med 352:2477-2486

10. Falavigna M, Schmidt MI, Trujillo J et al (2012) Effectiveness of gestational diabetes treatment: a systematic review with quality of evidence assessment. Diabetes Res Clin Pract 98:396-405

11. Horvath K, Koch K, Jeitler K, et al (2010) Effects of treatment in women with gestational diabetes mellitus: systematic review and meta-analysis. BMJ 340

12. Landon MB, Spong CY, Thom E et al (2009) A multicenter, randomized trial of treatment for mild gestational diabetes. N Engl $\mathrm{J}$ Med 361:1339-1348

13. Lohse N, Marseille E, Kahn JG (2011) Development of a model to assess the cost-effectiveness of gestational diabetes mellitus screening and lifestyle change for the prevention of type 2 diabetes mellitus. Int J Gynecol Obstet 115(Supplement 1):S20-S25

14. Mission JF, Ohno MS, Cheng YW, Caughey AB (2012) Gestational diabetes screening with the new IADPSG guidelines: a costeffectiveness analysis. Am J Obstet Gynecol 207:326.e321326.e329

15. Nicholson WK, Fleisher LA, Fox HE, Powe NR (2005) Screening for gestational diabetes mellitus: a decision and cost-effectiveness analysis of four screening strategies. Diabetes Care 28:1482-1484
16. Round JA, Jacklin P, Fraser RB, Hughes RG, Mugglestone MA, Holt RIG (2011) Screening for gestational diabetes mellitus: costutility of different screening strategies based on a woman's individual risk of disease. Diabetologia 54:256-263

17. Werner EF, Pettker CM, Zuckerwise L et al (2012) Screening for gestational diabetes mellitus: are the criteria proposed by the International Association of the Diabetes and Pregnancy Study Groups cost-effective? Diabetes Care 35:529-535

18. Falavigna M, Prestes I, Schmidt MI, Duncan BB, Colagiuri S, Roglic G (2013) Impact of gestational diabetes mellitus screening strategies on perinatal outcomes: a simulation study. Diabetes Res Clin Pract 99:358-365

19. Cullinan J, Gillespie P, Owens L, Dunne F (2012) Accessibility and screening uptake rates for gestational diabetes mellitus in Ireland. Health Place 18:339-348

20. Tierney M, O’Dea A, Glynn L, Carmody L, McGuire B, Dunne F (2014) The prevalence rate and rate of uptake of screening for gestational diabetes mellitus (GDM) in primary versus secondary care. Irish J Med Sci S447

21. Tierney M, O’Dea A, Danyliv A, Glynn L, McGuire B, Dunne F (2014) A qualitative study of the health behaviours of women after gestational diabetes (GDM). In: American Diabetes Association 74th Scientific Sessions. Diabetes, San Francisco

22. Health Information and Quality Authority (2014) Guidelines for the economic evaluation of health technologies in Ireland. Available from www.hiqa.ie/publications/guidelines-economic-evaluationhealth-technologies-ireland, accessed 20 Sep 2015

23. Curtis L (2014) Unit costs of health and social care 2014. Personal social services research unit. University of Kent, Canterbury

24. OECD (2014) PPPs and exchange rates. Available from http://stats. oecd.org/Index.aspx?datasetcode=SNA_TABLE4\#, accessed 20 Sept 2015

25. Gillespie P, O’Neill C, Avalos G, O'Reilly M, Dunne F, for the ADIPC (2011) The cost of universal screening for gestational diabetes mellitus in Ireland. Diabet Med 28:912-918

26. Casemix/HIPE (2014) Irish Casemix programme. www.hiqa.ie/ healthcare/health-information/data-collections/online-catalogue/ irish-casemix-programme, accessed 20 Sept 2015

27. Pourat N, Martinez A, Jones J, Gregory K, Korst L, Kominski G (2013) Costs of gestational hypertensive disorders in California: hypertension, preeclampsia, and eclampsia. UCLA Center for Health Policy Research, Los Angeles

28. Shmueli A, Meiri H, Gonen R (2012) Economic assessment of screening for pre-eclampsia. Prenat Diagn 32:29-38

29. Meads CA, Cnossen J, Meher S et al (2008) Methods of prediction and prevention of pre-eclampsia: systematic reviews of accuracy and effectiveness literature with economic modelling. Health Technol Assess 12:1-270

30. Barton JR, Istwan NB, Rhea D, Collins A, Stanziano GJ (2006) Cost-savings analysis of an outpatient management program for women with pregnancy-related hypertensive conditions. Dis Manag 9:236-241

31. Mistry H, Heazell AEP, Vincent O, Roberts T (2013) A structured review and exploration of the healthcare costs associated with stillbirth and a subsequent pregnancy in England and Wales. BMC Pregnancy Childbirth 13:236

32. Mangham LJ, Petrou S, Doyle LW, Draper ES, Marlow N (2009) The cost of preterm birth throughout childhood in England and Wales. Pediatrics 123:e312-e327

33. Culligan P, Myers J, Goldberg R, Blackwell L, Gohmann S, Abell T (2005) Elective cesarean section to prevent anal incontinence and brachial plexus injuries associated with macrosomia - a decision analysis. Int Urogynecol J 16:19-28

34. CSO (2009). Irish Life Tables. Central Statistics Office (CSO) Cork, Ireland 
35. Kuppermann M, Nease RF Jr, Learman LA, Gates E, Blumberg B, Washington AE (2000) Procedure-related miscarriages and down syndrome-affected births: implications for prenatal testing based on women's preferences. Obstet Gynecol 96:511-516

36. Mautner E, Greimel E, Trutnovsky G, Daghofer F, Egger JW, Lang U (2009) Quality of life outcomes in pregnancy and postpartum complicated by hypertensive disorders, gestational diabetes, and preterm birth. J Psychosom Obstet Gynecol 30:231-237

37. O'Dea A, Infanti JJ, Gillespie P et al (2014) Screening uptake rates and the clinical and cost effectiveness of screening for gestational diabetes mellitus in primary versus secondary care: study protocol for a randomised controlled trial. Trials 15:1-9

38. Layte R (2009) Projecting the impact of demographic change on the demand for and delivery of healthcare in Ireland. ESRI, Dublin

39. Vääräsmäki M, Pouta A, Elliot P et al (2009) Adolescent manifestations of metabolic syndrome among children born to women with gestational diabetes in a general-population birth cohort. Am J Epidemiol 169:1209-1215
40. Clausen TD, Mathiesen ER, Hansen T et al (2009) Overweight and the metabolic syndrome in adult offspring of women with diettreated gestational diabetes mellitus or type 1 diabetes. J Clin Endocrinol Metab 94:2464-2470

41. Krakowiak P, Walker CK, Bremer AA et al (2012) Maternal metabolic conditions and risk for autism and other neurodevelopmental disorders. Pediatrics 129:e1121-e1128

42. Boney CM, Verma A, Tucker R, Vohr BR (2005) Metabolic syndrome in childhood: association with birth weight, maternal obesity, and gestational diabetes mellitus. Pediatrics 115:e290-e296

43. Harder T, Rodekamp E, Schellong K, Dudenhausen JW, Plagemann A (2007) Birth weight and subsequent risk of type 2 diabetes: a meta-analysis. Am J Epidemiol 165:849-857

44. Wang X, Liang L, Junfen FU, Lizhong DU (2007) Metabolic syndrome in obese children born large for gestational age. Indian $\mathrm{J}$ Pediatr 74:561-565

45. Harder T, Roepke K, Diller N, Stechling Y, Dudenhausen JW, Plagemann A (2009) Birth weight, early weight gain, and subsequent risk of type 1 diabetes: systematic review and meta-analysis. Am J Epidemiol 169:1428-1436 